\title{
Multi-Frequency Contrast Source Inversion for Reflection Seismic Data
}

\author{
Shoudong Wang ${ }^{1,2,3, *}$ and Ru-Shan $\mathrm{Wu}^{3}$ \\ 1 State Key Laboratory of Petroleum Resources and Prospecting, China University of \\ Petroleum (Beijing), Beijing 102249, P.R. China. \\ 2 National Engineering Laboratory of Offshore Oil Exploration, China University of \\ Petroleum (Beijing), Beijing 102249, P.R. China. \\ 3 Modeling and Imaging Laboratory, Earth and Planetary Sciences, University of \\ California, Santa Cruz, CA 95064, USA.
}

Received 12 April 2018; Accepted (in revised version) 16 December 2018

\begin{abstract}
In the contrast source inversion (CSI) method, the contrast sources (equivalent scattering sources) and the contrast (parameter perturbation) are iteratively reconstructed by an alternating optimization scheme. Traditionally integral equation CSI method is formulated for transmission tomography using analytic Green's function in homogeneous background. To extend the method to the case of reflection seismology, in this paper, we use WKBJ method to compute the Green's function of depth dependent background media and the solving method of equations to initialize the contrast source of different frequencies, resulting in an efficient method to invert multifrequency reflection seismic data - multi-frequency contrast source inversion method (MFCSI). Numerical results for the Marmousi model show that MFCSI method can obtain good results even when low frequency data are missing, in which case the conventional FWI fails.
\end{abstract}

AMS subject classifications: 35L05, 35A35, 58J45

Key words: Contrast source inversion, full waveform inversion, frequency domain inversion, integral equation CSI.

\section{Introduction}

Full waveform inversion (FWI) was introduced to the exploration seismology community by Lailly in [12] and Tarantola in [18]. They regarded seismic inversion as a minimization of the misfit between recorded and modeled data. In [5], Bunks et al. developed FWI in the time domain and proposed successive inversion of multi-frequency band inversion. Pratt et al. studied the FWI in frequency domain in [15] and Shin extended FWI to the

${ }^{*}$ Corresponding author. Email addresses: ctlab@cup.edu.cn (S. Wang), rwu@ucsc.edu (R.-S. Wu) 
Laplace domain in [16]. Shin and Cha combined the frequency domain and Laplace domain and studied the FWI method in the Laplace-Fourier domain in [17]. Readers can be referred to [20] for a detailed review. On the other hand, the FWI can be considered as an inverse scattering problem. The inverse scattering problem has been studied for many years and it has numerous applications. For seismic imaging and inversion, see [22] for a review. In addition the approach has been developed in other fields, such as target identification, non-destructive testing and medical imaging. In [21], Wang and Chew (1989) proposed Born iterative method (BIM) where the Green's function remains unchanged in the iterative procedures for solving inverse scattering problem. In [6], Chew and Wang (1990) proposed the distorted Born iterative method (DBIM), where the Green's function is updated in every iteration step. In [11], Kleinman and van den Berg (1992) introduced the modified gradient method where the cost functional consists of the superposition of the mismatch of the measured field data with the calculated scattered field and the error in satisfying the state equation. The modified gradient method formed the base of the contrast source inversion (CSI) method in [19]. In the CSI method, the contrast sources (the product of contrast and total wavefield) and the contrast (perturbation) itself, are iteratively reconstructed by an alternately updating method. This is in contrast to the modified gradient method, where the fields and the contrast are updated simultaneously. The CSI method outperforms the modified gradient method, and is computationally faster and uses less memory. In [1], Abubakar et al. introduced the finite difference contrast source inversion (FDCSI) method. Unlike the CSI method using the integral equation (IE) approach, it uses a finite difference (FD) approach as its backbone and can readily employ an arbitrary inhomogeneous medium as its background media. In [2], Abubakar et al. applied the finite difference contrast source inversion method to seismic full waveform inversion problems and extended it to three dimensional geometry in [3]. In [4] Barrière et al. applied CSI method to relatively high contrast objects, in which situation the additive regularization can get better result. CSI method bears strong relationship with the T-matrix based inversion method $[8-10,13]$. However, the perturbation and Tmatrix updating in the above approach was realized by matrix operation, and the CSI method is still based on the least-square error minimization.

The traditional integral equation CSI method is commonly used in tomography, where the source and receiver positions locate in the opposite sides of the object and usually only single frequency data are used in the inversion [19]. For the reflection seismology, the source and receiver positions all locate on the earth's surface, which makes the inversion strong nonlinear and more difficult. Multiple frequency data must be used in the inversion in this case. For reflection geometry, data with different frequencies correspond to different wavenumber of the medium perturbation. In addition, a variable background medium must be used. Based on these features, we expand the CSI method and make it suitable for reflection seismic data. For the variable background we use the WKBJ method in [7] to compute the Green's function. Numerical results show that the multi-frequency (MFCSI) method can achieve better results than the conventional gradient method, especially for the case of data missing low frequency. 


\section{The acoustic scattering model}

Consider the following variable coefficient acoustic equation

$$
\Delta^{2} u=\frac{1}{v^{2}(r)} \frac{\partial^{2} u(r, t)}{\partial t^{2}}+f(r, t),
$$

where $v(r)$ is the velocity of the inhomogeneous media, $f(r, t)$ is the source function. $r$ represents the point in the space. $\Delta^{2}$ is Laplace operator and

$$
\Delta^{2} u=\frac{\partial^{2} u}{\partial x^{2}}+\frac{\partial^{2} u}{\partial z^{2}}
$$

Make Fourier transform on both side of (2.1) with respect to $t$, we get

$$
\left(\Delta^{2}+\frac{\omega^{2}}{v^{2}(r)}\right) u(r, \omega)=f(r, \omega)
$$

here $\frac{1}{v^{2}(r)}$ is the squared wave slowness, which can be expressed as

$$
\frac{1}{v^{2}(r)}=\frac{1}{v_{0}^{2}(r)}-\alpha(r)
$$

where $v_{0}(r)$ is the background velocity, which generally has very simple structure, such as constant velocity, constant gradient velocity. $\alpha(r)$ is the contrast function. Substituting (2.3) into (2.2), we get

$$
\left(\Delta^{2}+k_{0}^{2}\right) u(r, \omega)=\omega^{2} \alpha(r) u(r, \omega)+f(r, \omega),
$$

where $k_{0}=\frac{\omega}{v_{0}}$. We use $u_{0}$ to represent the wave field in background medium and

$$
\left(\Delta^{2}+k_{0}^{2}\right) u_{0}(r, \omega)=f(r, \omega) .
$$

Define the scattering field

$$
u_{s}(r, \omega)=u(r, \omega)-u_{0}(r, \omega) .
$$

Eq. (2.4) minus Eq. (2.5), we get

$$
\left(\Delta^{2}+k_{0}^{2}\right) u_{s}(r, \omega)=\omega^{2} \alpha(r) u(r, \omega) .
$$

This is the Helmholtz equation. We use $G\left(r, r^{\prime}, \omega\right)$ to represent its Green's function and

$$
\left(\Delta^{2}+k_{0}^{2}\right) G\left(r, r^{\prime}, \omega\right)=\delta\left(r-r^{\prime}\right) .
$$

With Green's Function, the solution of Eq. (2.7) can be written as

$$
u_{s}(r, \omega)=\int \omega^{2} \alpha\left(r^{\prime}\right) G\left(r, r^{\prime}, \omega\right) u\left(r^{\prime}, \omega\right) d r^{\prime} .
$$




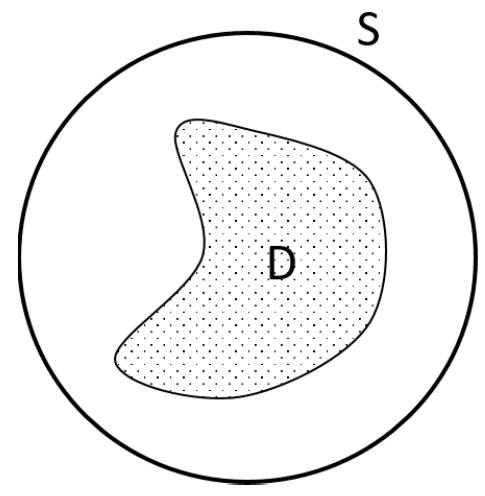

(a)

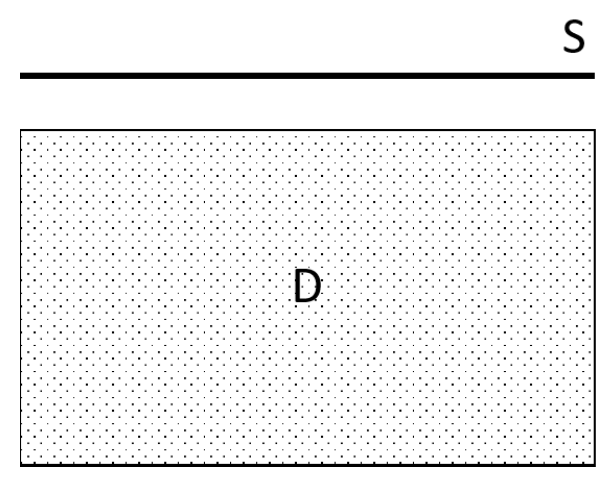

(b)

Figure 1: Data acquisition model. (a)Tomography; (b) Reflection seismic.

Add $u_{0}(r, \omega)$ on both side of Eq. (2.9), we get

$$
u(r, \omega)=u_{0}(r, \omega)+\omega^{2} \int \alpha\left(r^{\prime}\right) G\left(r, r^{\prime}, \omega\right) u\left(r^{\prime}, \omega\right) d r^{\prime} .
$$

As $\alpha(r)$ is given, Eq. (2.10) is the second kind of Fredholm integral equation. Solving the equation we can get wave field $u(r, \omega)$ and this is the forward problem.

In the specific case, we consider data acquisition model in Fig. 1(a). The perturbation is only in area D and the data is collected on the surface S. Then Eq. (2.9) become

$$
u_{s}(r, \omega)=\omega^{2} \int_{D} \alpha\left(r^{\prime}\right) G\left(r, r^{\prime}, \omega\right) u\left(r^{\prime}, \omega\right) d r^{\prime} \quad r \in S
$$

and (2.10) become

$$
u(r, \omega)=u_{0}(r, \omega)+\omega^{2} \int_{D} \alpha\left(r^{\prime}\right) G\left(r, r^{\prime}, \omega\right) u\left(r,{ }^{\prime} \omega\right) d r^{\prime} \quad r \in D .
$$

The forward problem is to find $u(r, \omega), r \in D$ and $u_{s}(r, \omega), r \in S$ by solving Eqs. (2.12) and (2.11). The inverse scattering problem is to find $\alpha(r)$ for given $u_{s}(r, \omega), r \in S$. Fig. 1(a) is the typical transmission tomography geometry. For reflection seismic, Fig. 1(b) shows the geometry.

\section{The CSI method}

As described above, the inverse scattering problem can be described by Eqs. (2.11) and (2.12). Background velocity can be assumed as constant and the Green's function in the 2D case can be computed by

$$
G\left(r, r^{\prime}\right)=(i / 4) H_{0}^{(1)}\left(k_{0}\left|r-r^{\prime}\right|\right),
$$


where $H_{0}^{(1)}$ is the zero-order Hankel function of the first kind. In general, the background velocity is not necessarily the homogeneous medium, but the Green's function should be able to be quickly calculated. Using the matrix formalism, Eq. (2.11) becomes

$$
\mathbf{U}_{s}=\mathbf{G}^{(s)} \mathbf{A} \mathbf{U}
$$

and Eq. (2.12) becomes

$$
\mathbf{U}=\mathbf{U}_{0}+\mathbf{G A U},
$$

where $\mathbf{A}$ is the contrast function, $\mathbf{U}$ is the total field vector, $\mathbf{U}_{0}$ is the background field vector, $\mathbf{U}_{s}$ is the scattered field vector and $\mathbf{G}^{(s)}$ denotes the Green's function for surface data calculation. Eqs. (3.1) and (3.2) are for the single source case. For multi-source case, Eqs. (3.1) and (3.2) become

$$
\begin{aligned}
& \mathbf{U}_{s, i}=\mathbf{G}_{i}^{(s)} \mathbf{A U}_{i}, \quad i=1,2, \cdots, N, \\
& \mathbf{U}_{i}=\mathbf{U}_{0, i}+\mathbf{G A U}_{i}, \quad i=1,2, \cdots, N,
\end{aligned}
$$

where $i$ represents the $i$-th shot, $N$ is the number of the shots. Eq. (3.3) is called data equation and (3.4) is the state equation. From Eq. (3.4), we can find a formal solution of $\mathbf{U}_{i}$,

$$
\mathbf{U}_{i}=(\mathbf{I}-\mathbf{G A})^{-\mathbf{1}} \mathbf{U}_{0, i}, \quad i=1,2, \cdots, N .
$$

Substituting it into the data equation we obtain

$$
\mathbf{U}_{s, i}=\mathbf{G}_{i}^{(s)}\left[\mathbf{A}(\mathbf{I}-\mathbf{G A})^{-\mathbf{1}} \mathbf{U}_{0, i}\right], \quad i=1,2, \cdots, N .
$$

Approximating the inverse operator by

$$
(\mathbf{I}-\mathbf{G A})^{-\mathbf{1}} \approx \mathbf{I}
$$

leads to the Born approximation. If the iterative methods is used, where a sequence $\left\{\mathbf{A}^{\mathbf{n}}\right\}$ is constructed, the approximation

$$
\left(\mathbf{I}-\mathbf{G A}^{\mathbf{n}}\right)^{-\mathbf{1}} \approx\left(\mathbf{I}-\mathbf{G A}^{\mathbf{n}-\mathbf{1}}\right)^{-\mathbf{1}}
$$

gives the iterative Born series method.

The CSI method is proposed by van den Berg in [19]. Its idea is to treat the product of contrast and field as one variable. Introducing the contrast source

$$
\mathbf{W}_{i}=\mathbf{A U}_{i}, \quad i=1,2, \cdots, N,
$$

data equation (3.3) becomes

$$
\mathbf{U}_{s, i}=\mathbf{G}_{i}^{(s)} \mathbf{W}_{i}, \quad i=1,2, \cdots, N,
$$


and Eq. (3.4) becomes

$$
\mathbf{W}_{i}=\mathbf{A U}_{0, i}+\mathbf{A G} \mathbf{W}_{i}, \quad i=1,2, \cdots, N .
$$

The inversion can be described as to find the A satisfying Eqs. (3.10) and (3.11). So we define the cost functional

$$
\sum_{i=1}^{N}\left\|\mathbf{G}_{i}^{(s)} \mathbf{W}_{i}-\mathbf{U}_{s, i}\right\|^{\mathbf{2}}+\beta \sum_{i=1}^{N}\left\|(\mathbf{I}-\mathbf{A G}) \mathbf{W}_{i}-\mathbf{A} \mathbf{U}_{0, i}\right\|^{\mathbf{2}},
$$

where $\beta$ is a weight coefficient, and minimizing the cost functional we can find the contrast A. The CSI method iteratively minimize the cost functional using an alternating method which first updates contrast source $\mathbf{W}_{i}$ and then updates contrast $\mathbf{A}$. Suppose we have obtained $\mathbf{W}_{i}^{n-1}$ and $\mathbf{A}^{n-1}$, The CSI method finds $\mathbf{W}_{i}^{n}$ and $\mathbf{A}^{n}$ in the following manner.

For the given $\mathbf{A}^{n-1}$ the cost functional (3.12) become

$$
\sum_{i=1}^{N}\left\|\mathbf{G}_{i}^{(s)} \mathbf{W}_{i}^{n-1}-\mathbf{U}_{s, i}\right\|^{2}+\beta \sum_{i=1}^{N}\left\|\left(\mathbf{I}-\mathbf{A}^{n-1} \mathbf{G}\right) \mathbf{W}_{i}^{n-1}-\mathbf{A}^{n-1} \mathbf{U}_{0, i}\right\|^{\mathbf{2}} .
$$

Minimizing this functional by the conjugate gradient method, we can obtain $\mathbf{W}_{i}^{n}$,

$$
\mathbf{W}_{i}{ }^{n}=\mathbf{W}_{i}^{n-1}+\gamma_{i}^{n} \mathbf{V}_{i}^{n},
$$

where $\mathbf{V}_{i}^{n}$ is the conjugate gradient direction and

$$
\begin{aligned}
& \mathbf{V}_{i}^{n}=\mathbf{g}_{i}^{n}+\frac{\left(\mathbf{g}_{i}^{n}\right)^{\mathbf{T}}\left(\mathbf{g}_{i}^{n}-\mathbf{g}_{i}^{n-1}\right)}{\left(\mathbf{g}_{i}^{n-1}\right)^{\mathbf{T}} \mathbf{g}_{i}^{n-1}} \mathbf{V}_{i}^{n-1}, \\
& \mathbf{g}_{i}^{n}=-\left(\mathbf{G}_{i}^{(s)}\right)^{\mathbf{T}}\left(\mathbf{G}_{i}^{(s)} \mathbf{W}_{i}^{n-1}-\mathbf{U}_{s, i}\right)-\beta\left(\mathbf{Q}^{n-1}\right)^{\mathbf{T}}\left(\mathbf{Q}^{n-1} \mathbf{W}_{i}^{n-1}-\mathbf{A}^{n-1} \mathbf{U}_{0, i}\right), \\
& \gamma_{i}^{n}=-\frac{\left(\mathbf{G}_{i}^{(s)} \mathbf{V}_{i}^{n}\right)^{\mathbf{T}}\left(\mathbf{G}_{i}^{(s)} \mathbf{W}_{i}^{n-1}-\mathbf{U}_{s, i}\right)+\beta\left(\mathbf{Q}^{n-1} \mathbf{V}_{i}^{n}\right)^{\mathbf{T}}\left(\mathbf{Q}^{n-1} \mathbf{W}_{i}^{n-1}-\mathbf{A}^{n-1} \mathbf{U}_{0, i}\right)}{\left(\mathbf{G}_{i}^{(s)} \mathbf{V}_{i}^{n}\right)^{\mathbf{T}}\left(\mathbf{G}_{i}^{(s)} \mathbf{V}_{i}^{n}\right)+\beta\left(\mathbf{Q}^{n-1} \mathbf{V}_{i}^{n}\right)^{\mathbf{T}}\left(\mathbf{Q}^{n-1} \mathbf{V}_{i}^{n}\right)}
\end{aligned}
$$

where $\mathbf{Q}^{n-1}=\left(\mathbf{I}-\mathbf{A}^{n-1} \mathbf{G}\right)$.

Once $\mathbf{W}_{i}^{n}$ is determined, $\mathbf{U}_{i}^{n}$ can be obtained via

$$
\mathbf{U}_{i}^{n}=\mathbf{U}_{0, i}+\mathbf{G} \mathbf{W}_{i}^{n} .
$$

And we can seek $\mathbf{A}^{n}$ to minimize the cost functional

$$
\sum_{i=1}^{N}\left\|\mathbf{U}_{i}^{n} \mathbf{A}^{n}-\mathbf{W}_{i}^{n}\right\|^{2}
$$


and

$$
\mathbf{A}^{n}=\left(\sum_{i=1}^{N}\left(\mathbf{U}_{i}^{n}\right)^{\mathbf{T}} \mathbf{U}_{i}^{n}\right)^{-\mathbf{1}} \sum_{i=1}^{N}\left(\mathbf{U}_{i}^{n}\right)^{\mathbf{T}} \mathbf{W}_{i}^{n} .
$$

This complete the description of the algorithm except for the starting values $\mathbf{W}_{i}^{0}$. We choose the starting value obtained by back propagation of observed data [19] and

$$
\mathbf{W}_{i}^{0}=\frac{\left\|\left(\mathbf{G}_{i}^{(s)}\right)^{\mathbf{T}} \mathbf{U}_{\mathbf{s}, \mathbf{i}}\right\|}{\left\|\mathbf{G}_{i}^{(s)}\left(\mathbf{G}_{i}^{(s)}\right)^{\mathbf{T}} \mathbf{U}_{\mathbf{s}, \mathbf{i}}\right\|}\left(\mathbf{G}_{i}^{(s)}\right)^{\mathbf{T}} \mathbf{U}_{\mathbf{s}, \mathbf{i}} .
$$

This complete the description of the CSI algorithm.

\section{The CSI method for reflection data}

In exploration seismology, velocity increases with depth. If we use the homogeneous background, the contrast will be quite large, and this will increase the nonlinearity and difficulty of inversion. Variable background medium must be employed for reflection seismic. We chose smooth, vertically varying background medium and use the WBKJ method to compute the Green's function of background medium. The WKBJ solutions [7] will be accurate if the wavelength of the waves is considerably shorter than the scale length of the variations in the medium and the computation efficiency of WKBJ method is high. This is the motivation for choosing the background parameters to be slowly varying. Other methods of calculating Green's function are also feasible, for example, the method of calculating the Green's function for an arbitrary heterogeneous reference medium numerically by solving the Lippmann-Schwinger equation $[9,10]$.

According to WKBJ method [7], for the vertically varying background medium, the 2D Green's function can be computed by

$$
G\left(x, z_{g} \mid 0, z^{\prime} ; \omega\right)=\frac{1}{2 \pi} \int d k_{x} e^{i k_{x} x} \frac{e^{i \int_{z^{\prime}}^{z g} d z q(z)}}{2 i \sqrt{q\left(z_{g}\right) q\left(z^{\prime}\right)}},
$$

where

$$
q(z)=\frac{\omega}{v_{0}(z)} \sqrt{1-\frac{k_{x}^{2} v_{0}^{2}(z)}{\omega^{2}}}
$$

$\left(0, z^{\prime}\right)$ is shot point coordinates, $\left(x, z_{g}\right)$ represents receiver coordinates and $v_{0}(z)$ is the background velocity.

In the conventional integral equation CSI method for transmission tomography, only one frequency is used in the inversion [19]. Multiple frequency data must be used in the inversion of the reflection seismic data. In $[2,3]$, Abubakar et al. extended the FDCSI to 
the reflection seismic data and also used multiple frequency data. The strategy of selecting frequency is similar to the gradient method (Gauss-Newton method) [14,15], which helps to mitigate the nonlinearity of the inversion problem. The CSI inversion method will encounter a problem when the inversion moves from one frequency to another frequency. In the inversion process, the CSI inversion method updates the contrast function, and also update contrast source iteratively. The contrast source changes when the data frequency changes and an initial value needs to be given. FDCSI does not have the problem because it changes the background for each frequency and calculates the Green's function by finite difference method. We use the inversion result of contrast function from the previous frequency as the initial value for the new frequency, say the $\mathrm{m}^{\text {th }}$ frequency, $A_{m}^{0}$. From (3.11), for the $i$-th shot, we can calculate the new initial contrast source by the following equation:

$$
\left(\mathbf{I}-\mathbf{A}_{m}^{0} \mathbf{G}^{m}\right) \mathbf{W}_{i, m}^{0}=\mathbf{A}_{m}^{0} \mathbf{U}_{0, i, m}
$$

Once we obtain the initial value $\mathbf{W}_{i, m}^{0}$, we can use formula (3.14) to update the $\mathbf{W}_{i, m}$ and use formula (3.20) to update the $\mathbf{A}_{m}$. The iterative process can run until the termination criterion is satisfied. This complete the description of the MFCSI algorithm.

\section{Discussion on computational complexity}

MFCSI method is separately carried out for each frequency data and the inverse process is from low frequency to high frequency. We just discuss the computational complexity of inversion for one frequency. Suppose the size of the model is $N_{z} \times N_{x}$, the number of unknowns is $N=N_{z} \times N_{x}$, the number of sources is $N_{s}$ and the number of receivers for each shot is $N_{r}$. The memory mainly needed for inversion is the Green's function matrix $\mathbf{G}$ in formula (3.13). The matrix $\mathbf{G}$ is $N \times N$ and it is dense complex matrix. The main computations of MFCSI are solving Eq. (4.2) and computing the gradient using formula (3.16). We use the LU decomposition method to solve Eq. (4.2). It can effectively save computations for the multi-source data and computations $=\mathcal{O}\left(\frac{1}{3} N^{3}+N_{s} * N^{2}\right)$. The computations of computing gradient are $\mathcal{O}\left(2 N_{s} *\left(N_{r} * N+N^{2}\right)\right)$.

\section{Numerical examples}

\subsection{Simple model}

The true velocity section of the first example is shown in the Fig. 2 and it is a very simple model. There are four homogeneous layers in the model. The model is $1000 \mathrm{~m}$ wide and $500 \mathrm{~m}$ deep. The target region, which starts at a depth of $100 \mathrm{~m}$, is discretized into $100 \times 40$ grid blocks that are $10 \mathrm{~m}$ in each direction. We employed 33 sources and 101 receivers located on the surface $z=0 \mathrm{~m}$. The lateral position of first shot is $x=10 \mathrm{~m}$ and 


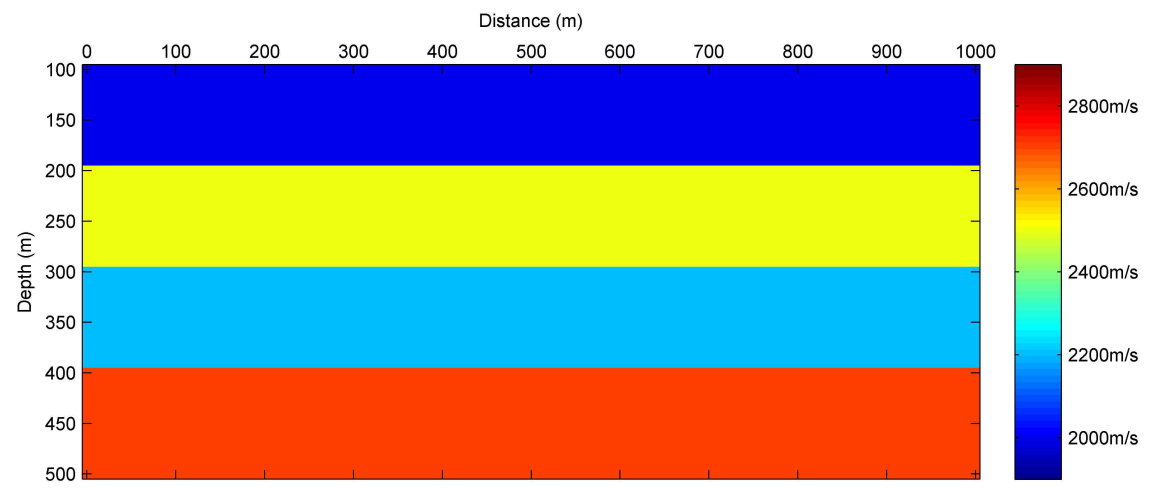

Figure 2: The true velocity model.

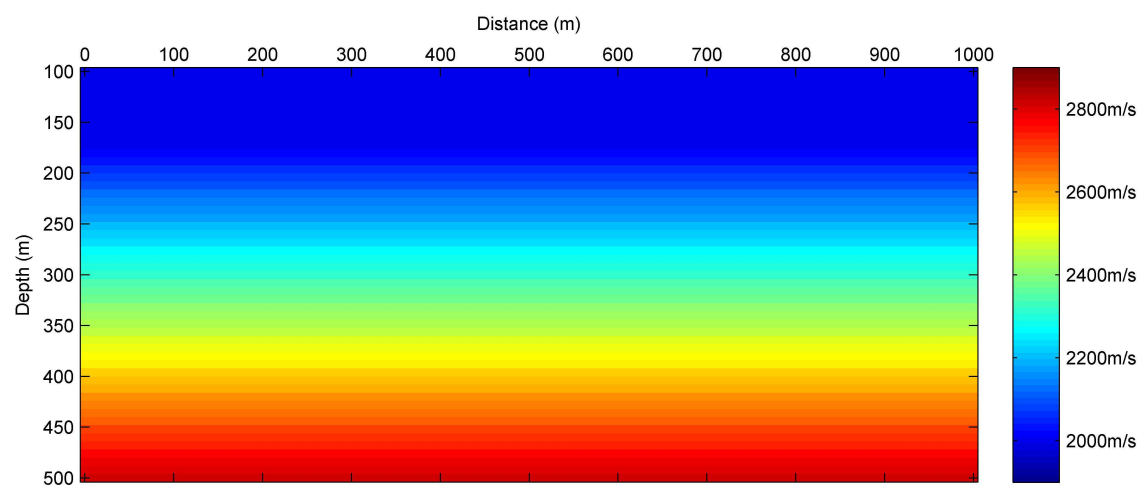

Figure 3: The background velocity.

the shot interval is $30 \mathrm{~m}$. The lateral position of first receiver is $x=0 \mathrm{~m}$ and receivers interval is $10 \mathrm{~m}$. For each shot, we use all the receivers. The background velocity we used is shown in Fig. 3. Based on experiences from many numerical experiments, we selected nine frequencies $(1,1.5,2.2,3.3,4.9,7.3,10.9,16.3$ and $24.4 \mathrm{~Hz})$ for inversion. We made inversion using each single frequency data and from low frequency to high frequency. The inverted velocity of low frequency data is used as the initial velocity of next inversion. The number of iterations of inversion for each frequency data is 60 . We first made the inversion using the conventional CSI method which use formula (3.21) to initialize the contrast source and the inversion results using the aforementioned nine frequencies is shown in Fig. 4. We can see that the conventional CSI method is not convergent and the inversion result is not acceptable. Fig. 5 shows the inversion results using the MFCSI method and Fig. 6 shows the comparison of true velocity, initial velocity and MFCSI inversion velocity at distance $200 \mathrm{~m}, 500 \mathrm{~m}$ and $800 \mathrm{~m}$. From the two figures, we can see that the MFCSI method can obtain good inversion result. 


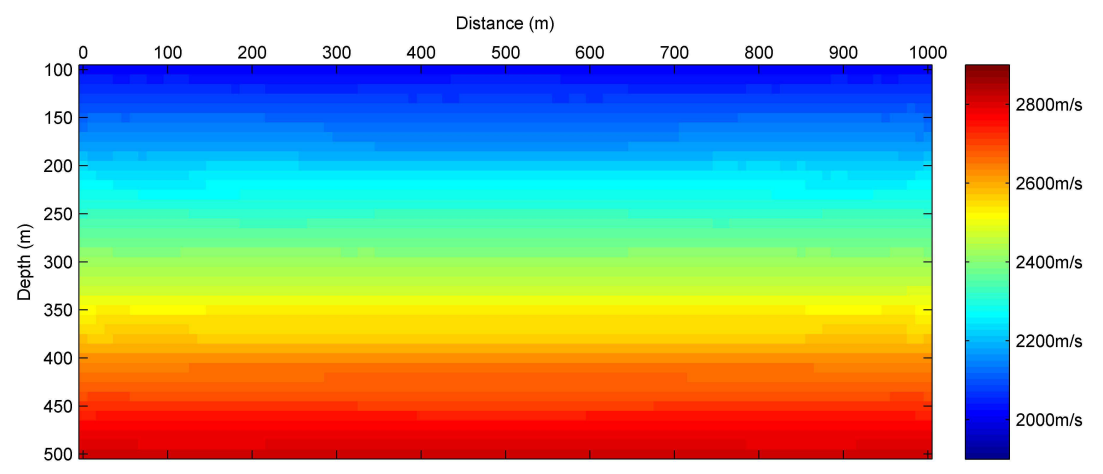

Figure 4: The inverted velocity model using the conventional CSI method.

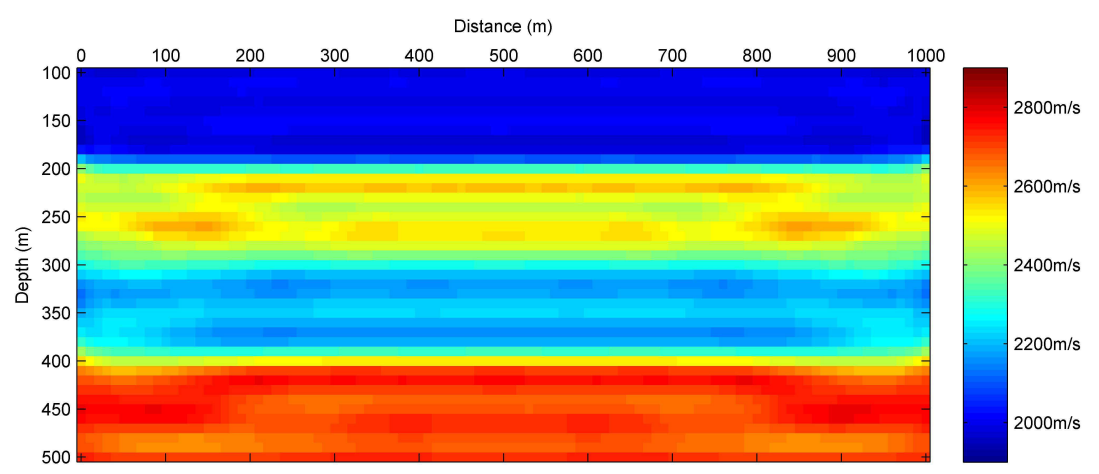

Figure 5: The inverted velocity model using the MFCSI method.

\subsection{Marmousi model}

The velocity model of the second example is a resampled 2D Marmousi model (Fig. 7) which is a more realistic structure. The model is $10000 \mathrm{~m}$ wide and $3080 \mathrm{~m}$ deep. The target region, which starts at a depth of $80 \mathrm{~m}$, is discretized into $250 \times 75$ grid blocks with size of $40 \mathrm{~m}$ in each direction (the original grid size is $4 \mathrm{~m}$ ). We employed 51 sources and 251 receivers located at surface $z=0 \mathrm{~m}$. The lateral position of first shot is $x=40 \mathrm{~m}$ and the shot interval is $200 \mathrm{~m}$. The lateral position of first receiver is $x=0 \mathrm{~m}$ and receivers interval is $40 \mathrm{~m}$. For each shot, we use all the receivers. Through averaging the true velocity in lateral and smoothing it in vertical, we got a depth-dependent smooth background velocity model (Fig. 8). We selected the nine frequencies $(1,1.5,2.2,3.3,4.9,7.3,10,14$ and $18 \mathrm{~Hz}$ ) for inversion and the inversion process is the same as the first example. Fig. 9 shows the inversion result using MFCSI method. The comparison of velocity profiles for the true velocity, initial velocity and MFCSI inversion velocity at distance $2000 \mathrm{~m}$, $4800 \mathrm{~m}$ and $7600 \mathrm{~m}$ is shown in Fig. 10. From the two figures, we can observe that the MFCSI method also can obtain good inversion result for the complex structure. 


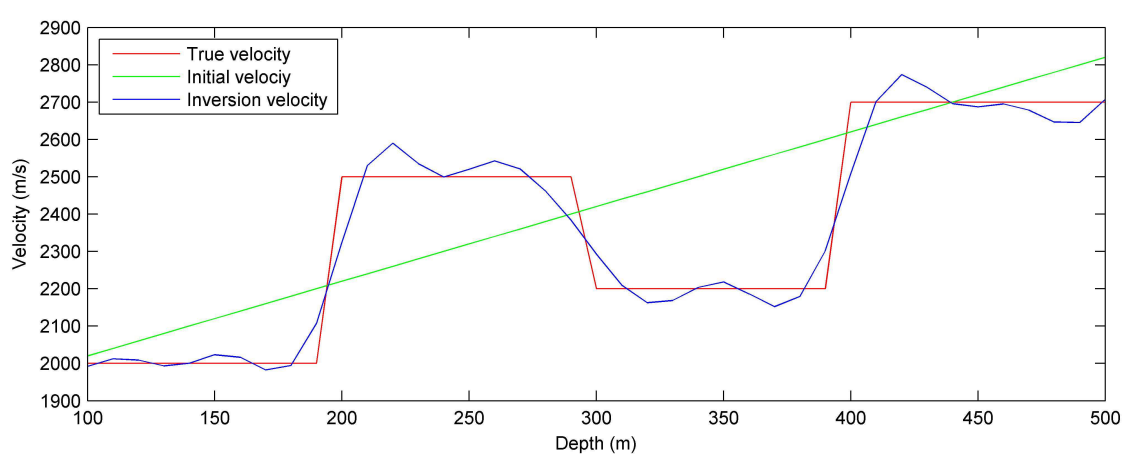

(a)

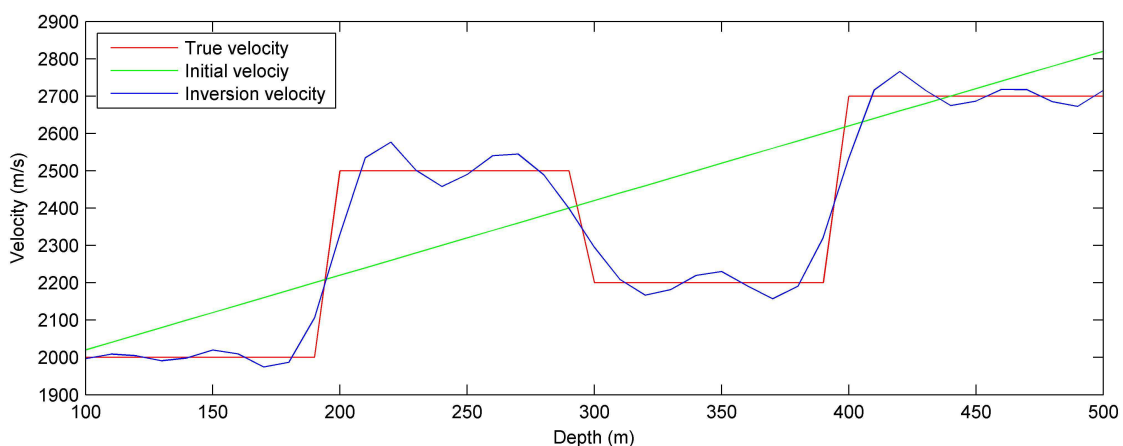

(b)

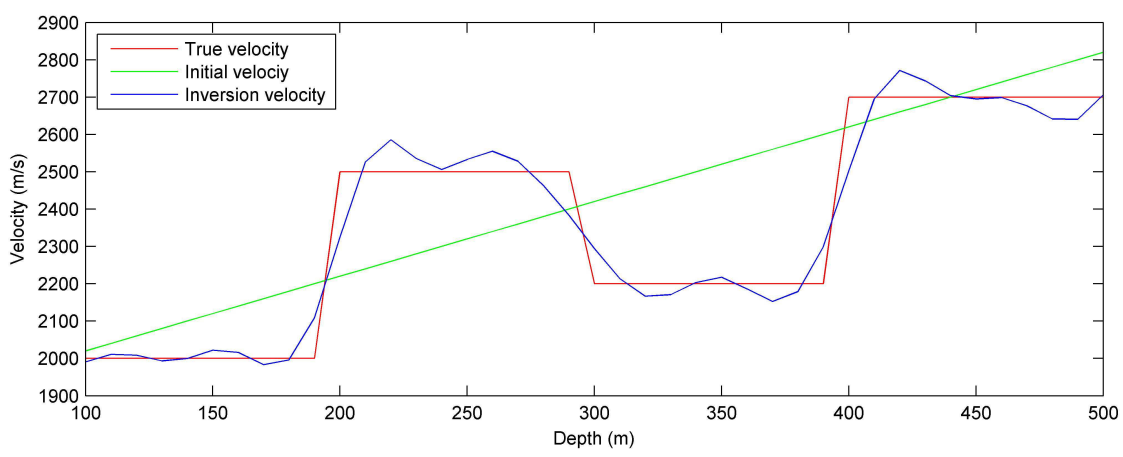

(c)

Figure 6: The comparison of true velocity, initial velocity and MFCSI inversion velocity at distance (a) $200 \mathrm{~m}$, (b) $500 \mathrm{~m}$ and (c) $800 \mathrm{~m}$.

In the above example, the background velocity model is obtained from the true velocity model. We know that, in the actual situation, we do not know the true velocity model. In addition, in seismic exploration there are no effective frequency component below 4 $\mathrm{Hz}$ in the real seismic data. In a more realistic example, we use a linear velocity as back- 


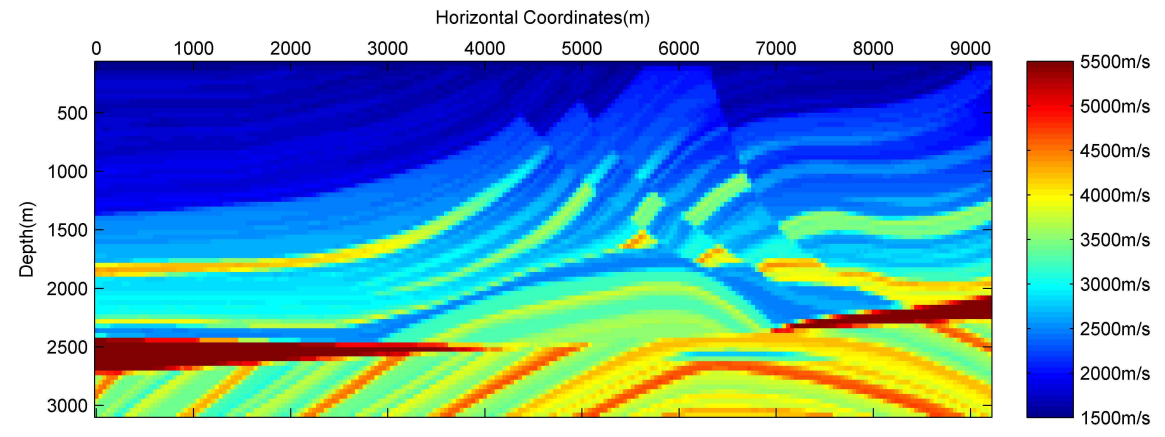

Figure 7: The resampled Marmousi velocity model.

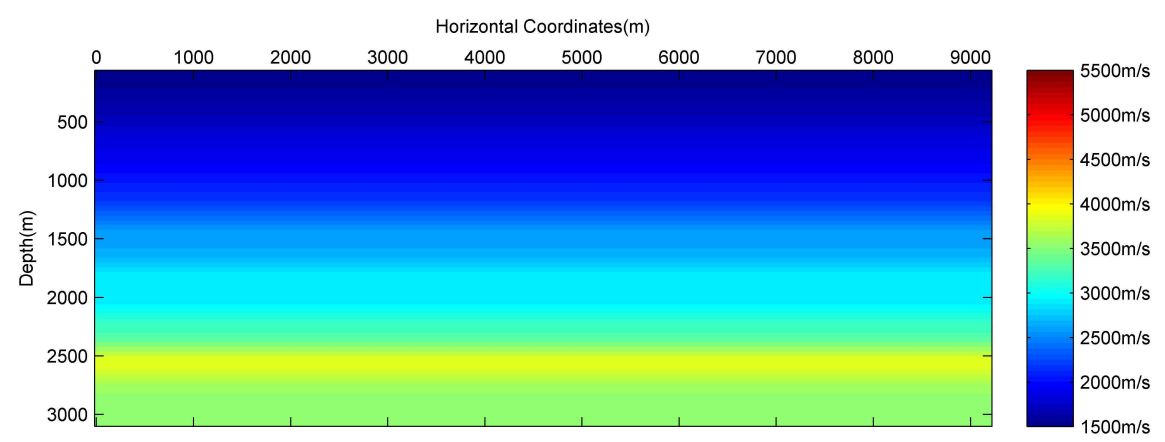

Figure 8: The depth dependent smooth background velocity model.

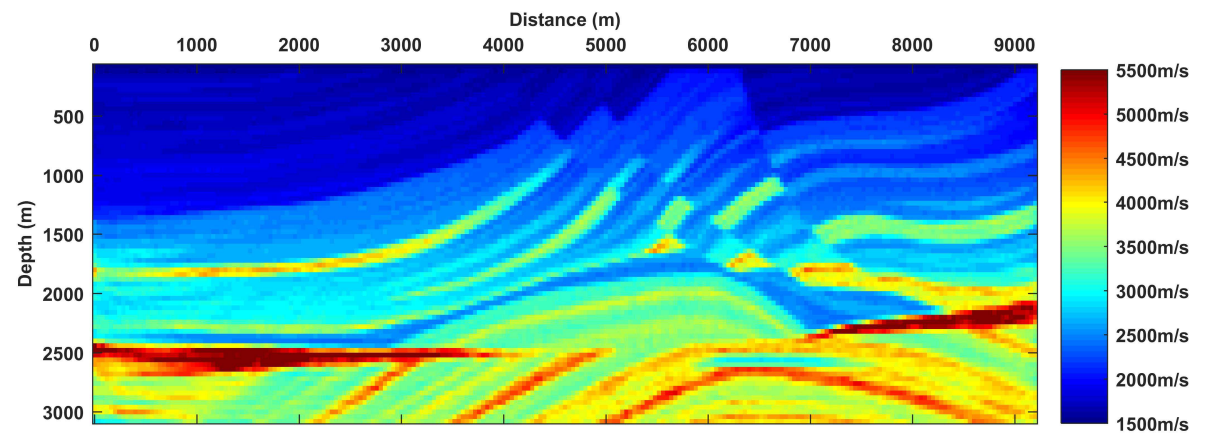

Figure 9: The MFCSI inversion velocity section.

ground velocity (Fig. 11) and do not use the lowest frequency data in the inversion. We selected nine frequencies $(4,4.25,4.5,4.75,5,7.5,11.25,14$ and $18 \mathrm{~Hz})$ for inversion. The other aspects in the inversion are the same as the second example. Fig. 12(b) shows the inversion result. For comparison, we also show the inversion result of using the fullband sources for the linear background medium (Fig. 12(a)). The comparison between 


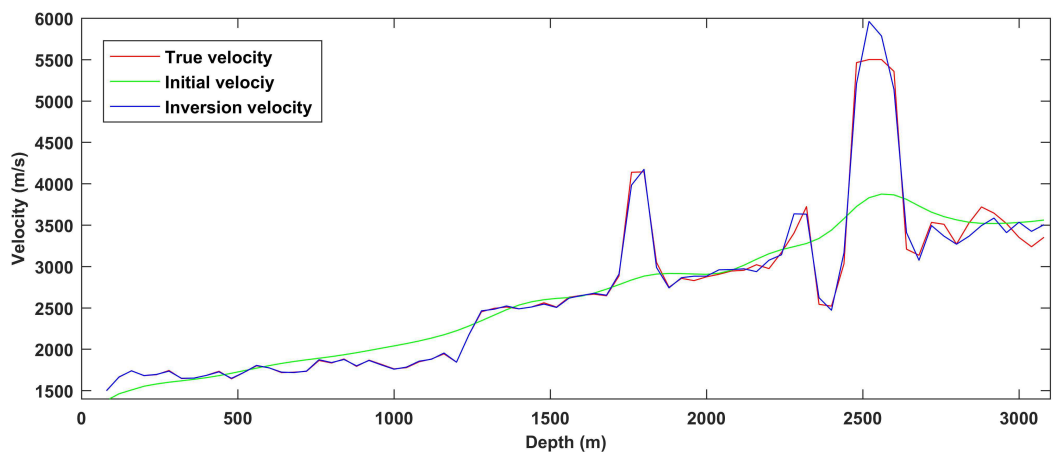

(a)

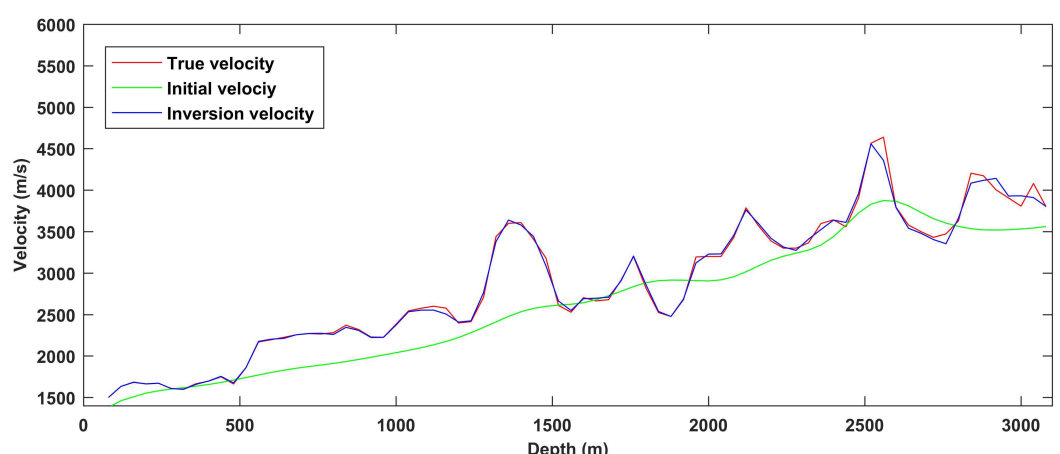

(b)

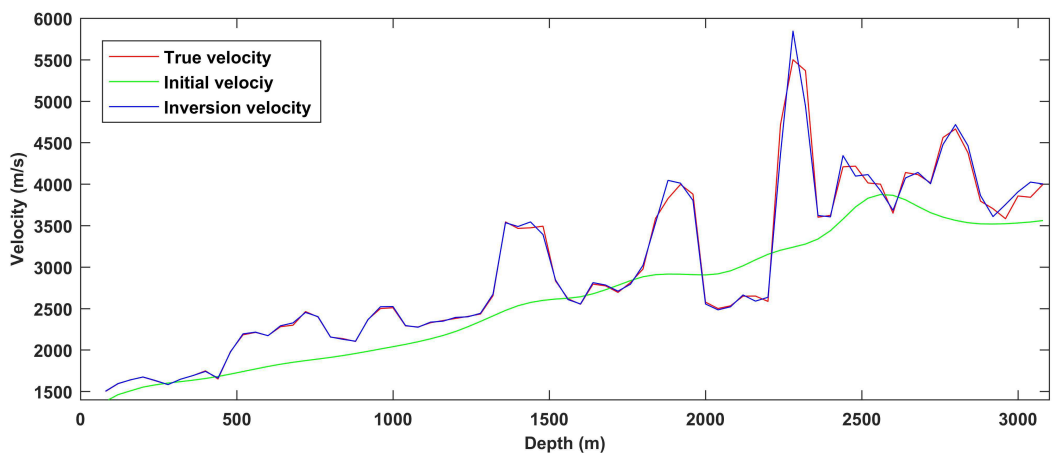

(c)

Figure 10: The comparison of true velocity, initial velocity and MFCSI inversion velocity at distance (a) $2000 \mathrm{~m}$, (b) $4800 \mathrm{~m}$ and (c) $7600 \mathrm{~m}$.

true velocity, initial velocity and MFCSI inversion velocity at distance $2000 \mathrm{~m}, 4800 \mathrm{~m}$ and $7600 \mathrm{~m}$ is shown in Fig. 13. From the two figures, we see that the MFCSI method also can obtain good inversion result using linear background velocity for the case of data missing low frequency. 


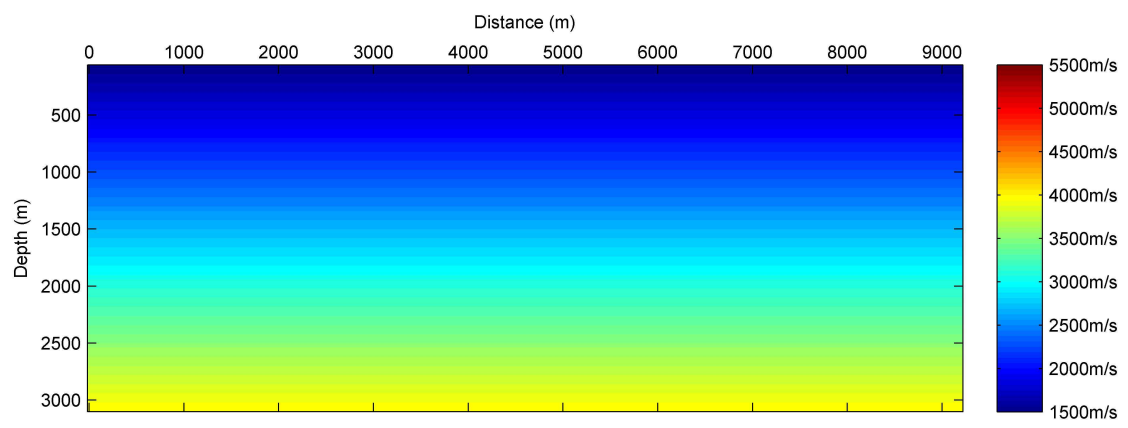

Figure 11: The linear background velocity model.

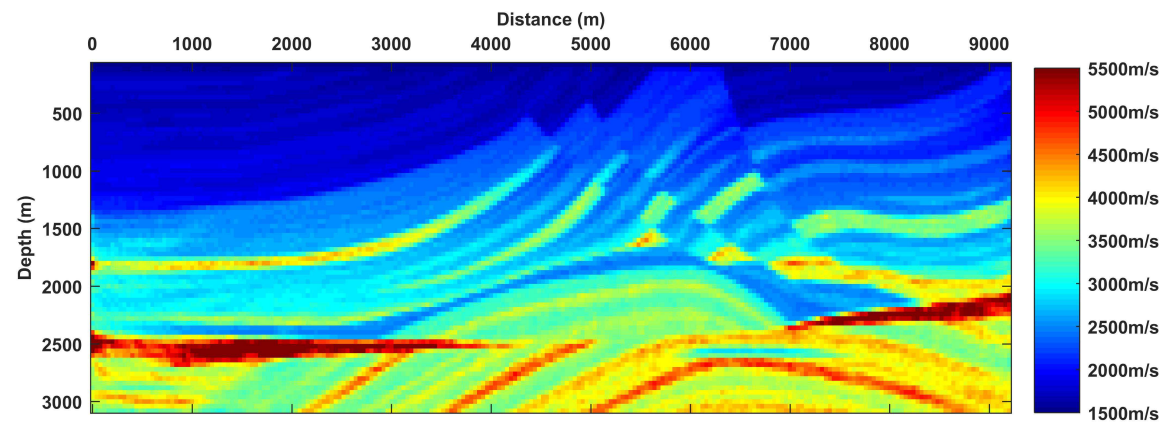

(a)

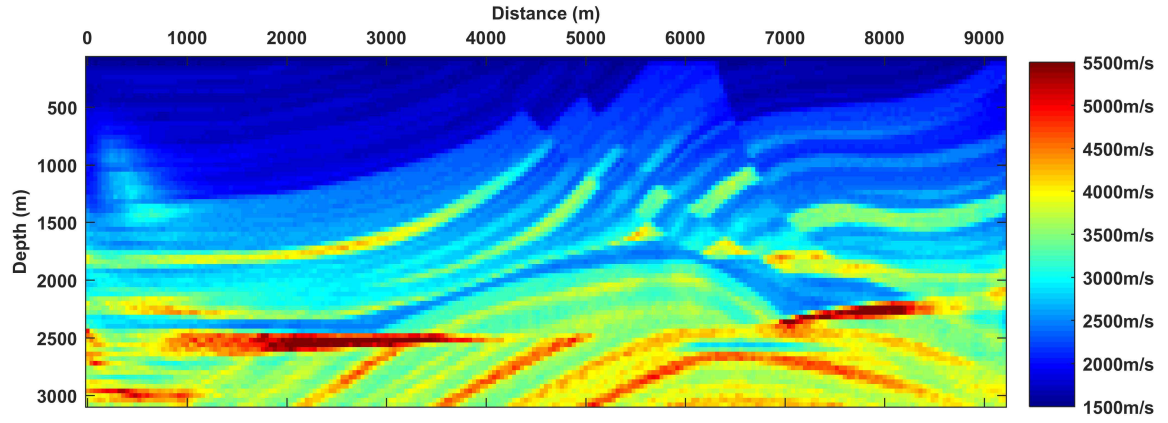

(b)

Figure 12: Inverted velocity model obtained using the MFCSI method with an initial velocity model where velocity increases linearly with depth. (a) Inversion results obtained by including data with frequency lower than $4 \mathrm{~Hz}$. (b) Inversion results obtained by excluding data with frequency lower than $4 \mathrm{~Hz}$.

To compare MFCSI method with the conventional gradient inversion method (GaussNewton method) $[5,15]$, we make the same model inversion using the gradient inversion method. The initial velocity model is a linear model shown in Fig. 11, same as the previ- 


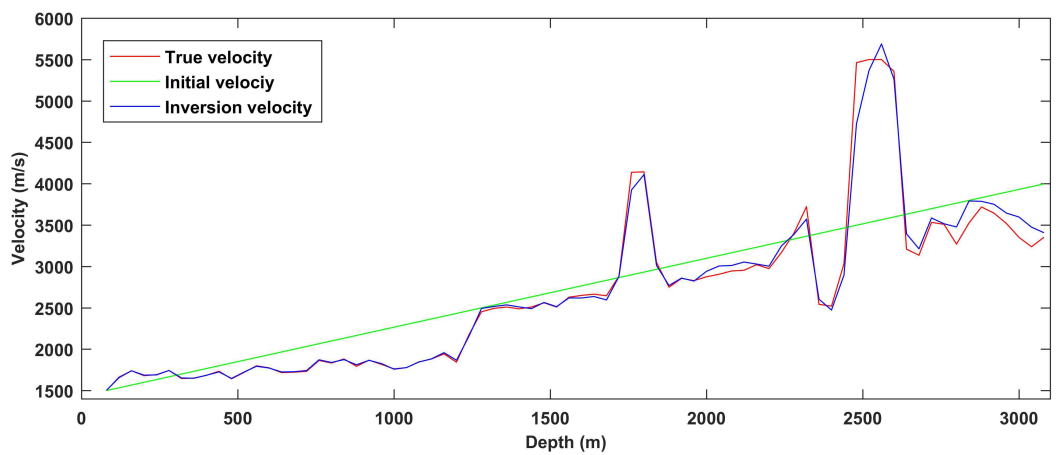

(a)

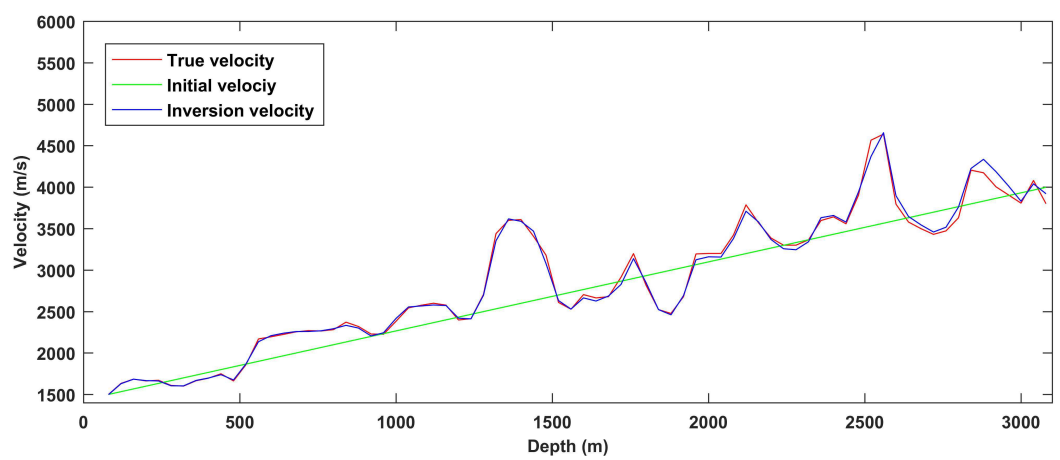

(b)

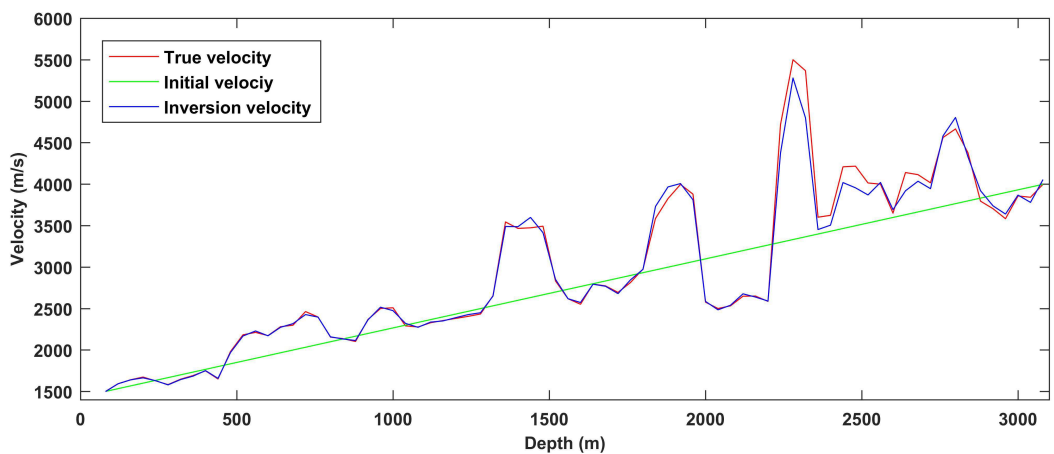

(c)

Figure 13: The comparison of true velocity, initial velocity and MFCSI inversion velocity at distance (a) $2000 \mathrm{~m}$, (b) $4800 \mathrm{~m}$ and (c) $7600 \mathrm{~m}$.

ous example. Fig. 14 shows the inversion result for full frequency band data $(1,1.5,2.2$, $3.3,4.9,7.3,10,14$ and $18 \mathrm{~Hz}$ ) and Fig. 15(a) is the inversion result for the case when inversion started from $3 \mathrm{~Hz}$ above, while Fig. 15(b) shows the result when inversion started from $4 \mathrm{~Hz}(4,4.25,4.5,4.75,5,7.5,11.25,14$ and $18 \mathrm{~Hz})$. From these comparisons, we can 


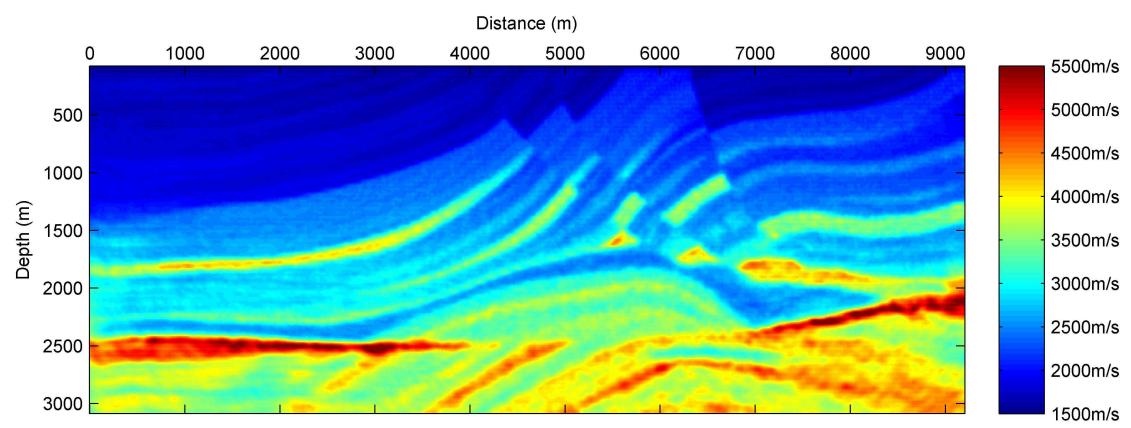

Figure 14: The inversion result using gradient method using the full frequency-band data.

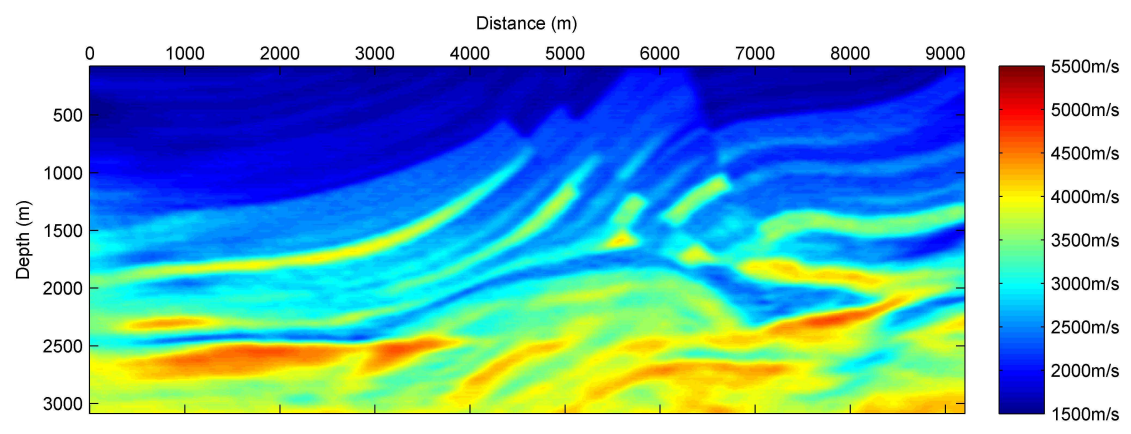

(a)

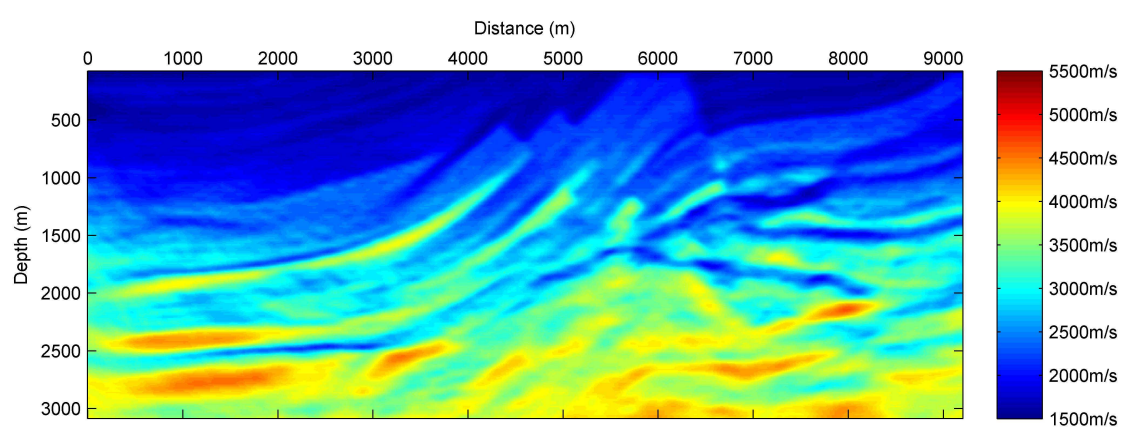

(b)

Figure 15: The inversion result using gradient method for data missing low frequency. (a) Data with frequency lower than $3 \mathrm{~Hz}$ are not used in the inversion. (b) Data with frequency lower than $4 \mathrm{~Hz}$ are not used in the inversion.

see that the gradient method can obtain good result for the full frequency band data and can give acceptable result when low-frequency data below $3 \mathrm{~Hz}$ are missing. However, the inversion result is much deteriorated and totally unacceptable when low frequency data below $4 \mathrm{~Hz}$ are cut. It shows that the nonlinearity is too strong in the latter case and 


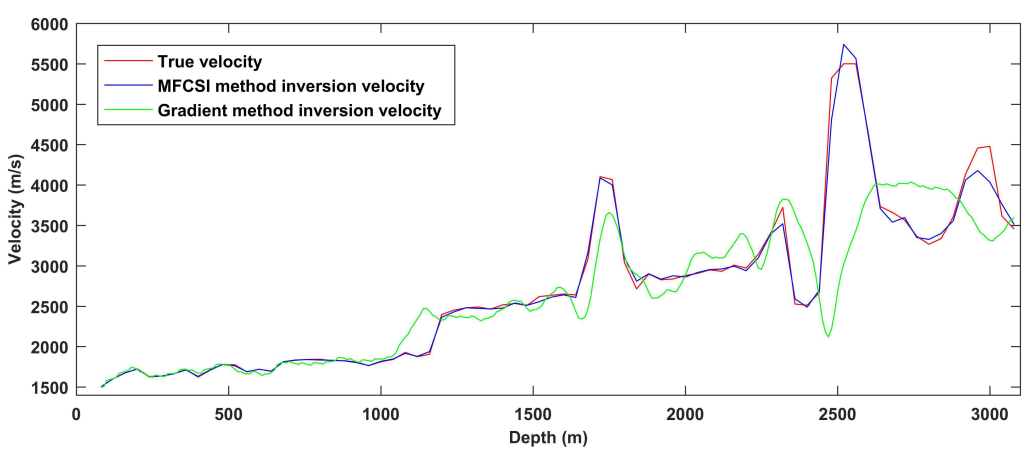

(a)

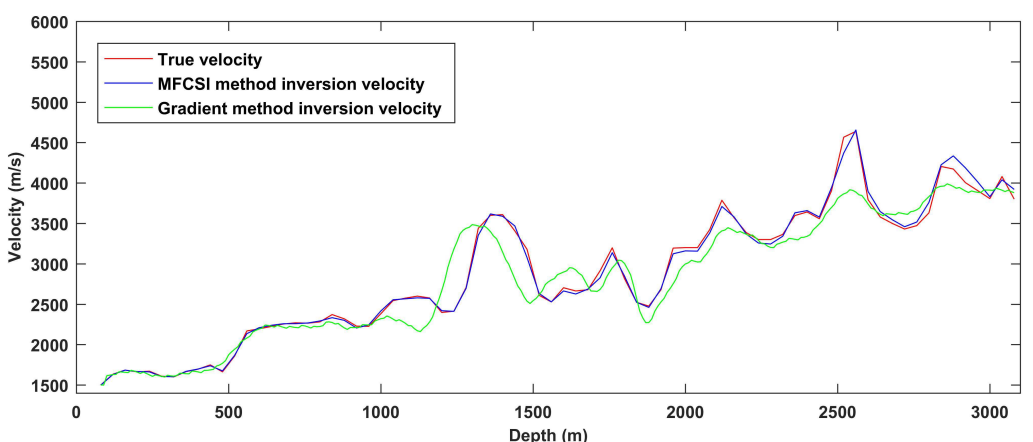

(b)

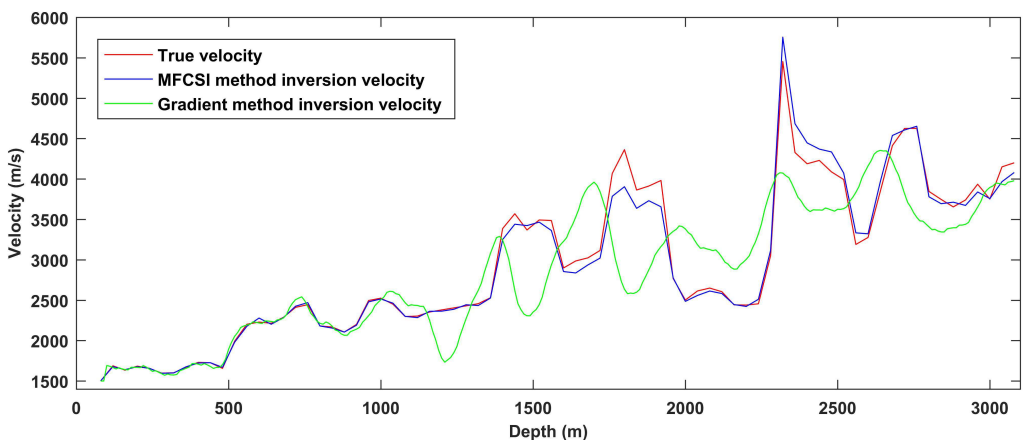

(c)

Figure 16: The comparison of inversion results using gradient method and MFCSI method for data missing low frequency at distance (Data with frequency lower than $4 \mathrm{~Hz}$ are not used in the inversion) (a) $2400 \mathrm{~m}$, (b) $4800 \mathrm{~m}$ and (c) $7200 \mathrm{~m}$.

the standard gradient method (Gauss-Newton or its variants) no longer functions properly for that case. In the meanwhile the MFCSI method can still work and give reasonable inversion result. Fig. 16 shows the comparison of inversion results using gradient method and MFCSI method for the low frequency missing (below $4 \mathrm{~Hz}$ ) data at distance $2400 \mathrm{~m}$, 


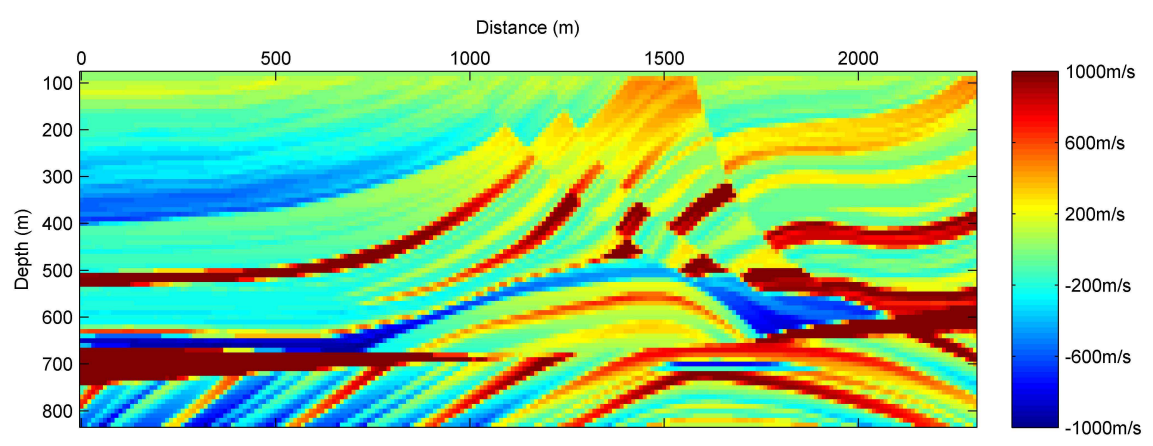

Figure 17: Difference of the true velocity and initial velocity.

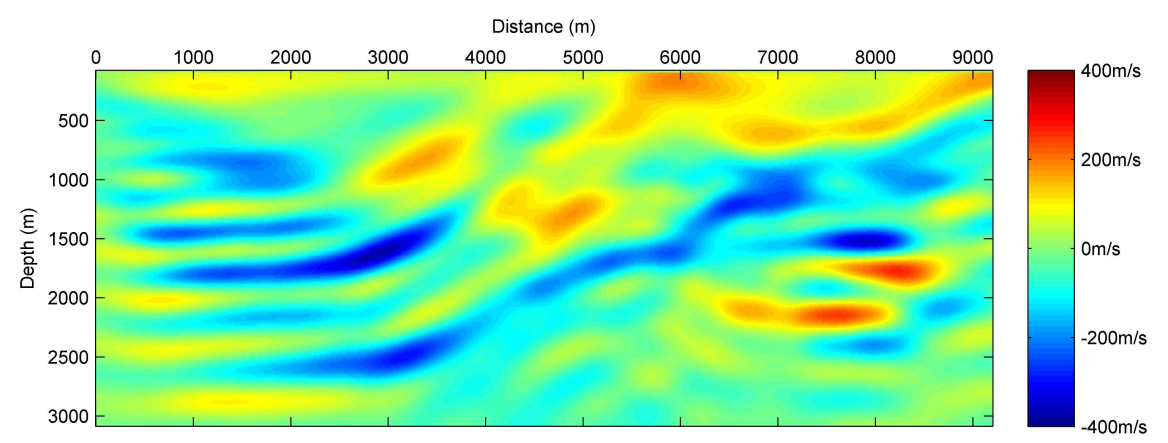

Figure 18: Velocity update of the first iteration using gradient method. Initial velocity is Fig. 11. Seismogram with frequency $4 \mathrm{~Hz}$ is used in the inversion.

$4800 \mathrm{~m}$ and $7200 \mathrm{~m}$. We can conclude that the MFCSI method has advantage over the gradient method for the case of data missing low frequency.

In order to further study the performance, we compare the velocity update in first iteration using gradient method and MFCSI. Fig. 17 shows the difference between true velocity and initial velocity. This figure can be used as a reference used to compare with different velocity updates. Fig. 18 is the velocity update using gradient method. Comparing Fig. 18 and Fig. 17, we can see that there are negative velocity updates in the shallow part of Fig. 18 which is not consistent with Fig. 17. Fig. 19 is the velocity update using MFCSI. Comparing Fig. 17, Fig. 18 and Fig. 19, we can see that the velocity update using MFCSI has much more long-wavelength components and is better than that of gradient method. Fig. 20 shows the reduction of data residuals with iterations. Compared with gradient method, the convergence of MFCSI method is faster and has avoided the false local minima. The gradient method only follows the update direction for data residual reduction. While CSI inversion is also constrained by the contrast errors in the model space. The key is the model reconstruction by the inversion of the lowest frequency. Contrast-source and contrast (perturbation) inversions have different difficulties. For contrast inversion, 


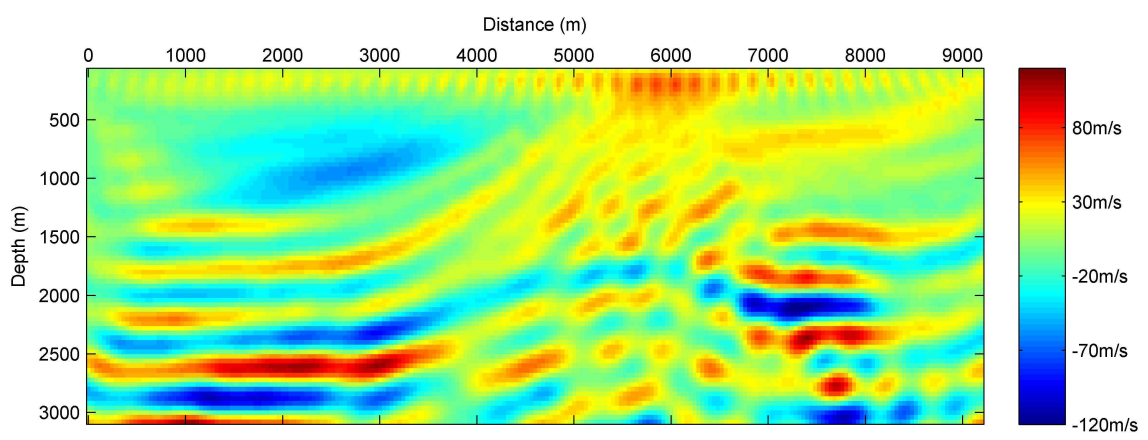

Figure 19: Velocity update of the first iteration using MFCSI method. Initial velocity is Fig. 11. Seismogram with frequency $4 \mathrm{~Hz}$ is used in the inversion.

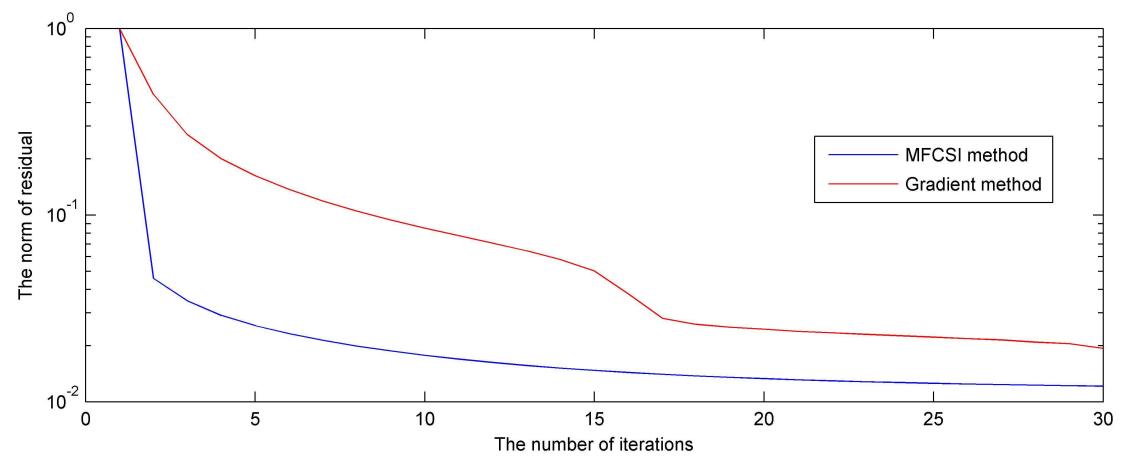

Figure 20: Reduction of data residuals with iterations. Comparison of MFCSI and gradient method.

the main problem is the strong nonlinearity. However, contrast source inversion itself is a linear inversion and its main difficulty is the nonuniqueness (illposedness) of inversion. Combining these two approaches together and taking advantages of both, MFCSI can work for stronger nonlinearity than the gradient inversion method.

\section{Conclusion}

We have improved the integral equation CSI method to apply to the inversion of multifrequency reflection seismic data. We use WKBJ method to compute the Green's function of depth dependent background media and equations solving method to initialize the contrast source for different frequencies. Two tests of this algorithm, performed with synthetic models, show that the multi-frequency CSI method can recover the velocity even for data missing low frequency. A comparison between the multi-frequency CSI method and conventional gradient method (Gauss-Newton method) shows that the multi-frequency CSI method produces better reconstruction of velocity than the conventional gradient method in the case where low-frequency data are not available. 


\section{Acknowledgments}

We thank Xiao-Bi Xie at University of California Santa Cruz (UCSC) for fruitful discussions and Guoxin Chen for providing the conventional gradient inversion results. This research is done when S. Wang is working in the Modeling and Imaging Laboratory, University of California, Santa Cruz, USA, as a visiting scholar. This research is supported by the National Science and Technology of Major Projects of China (grant no. 2016ZX05024-001-004) and the WTOPI Research Consortium of Modeling and Imaging Laboratory, University of California Santa Cruz, US.

\section{References}

[1] A. Abubakar, W. Hu, P. M. van den Berg and T. M. Habashy, A finite-difference contrast source inversion method. Inverse Problems, 24 (2008), 065004.

[2] A. Abubakar, W. Hu, T. M. Habashy and P. M. van den Berg, Application of the finitedifference contrast-source inversion algorithm to seismic full-waveform data. Geophysics, 74 (2009), WCC47-WCC58.

[3] A. Abubakar, G. Pan, M. Li, L. Zhang, T. M. Habashy and P. M. van den Berg, Threedimensional seismic full-waveform inversion using the finite-difference contrast source inversion method. Geophysical Prospecting, 59 (2011), 874-888.

[4] P. A. Barrire, J. Idier, J. J. Laurin, and Y. Goussard, Contrast source inversion method applied to relatively high contrast objects. Inverse Problems, 27 (2011), 075012.

[5] C. Bunks, F. M. Saleck, S. Zaleski and G. Chavent, Multiscale seismic waveform inversion. Geophysics, 60 (1995), 1457-1473.

[6] W. C. Chew, and Y. M. Wang, Reconstruction of two-dimensional permittivity distribution using the distorted Born iterative method. Medical Imaging, IEEE Transactions on, 9 (1990), 218-225.

[7] R. W. Clayton and R. H. Stolt, A Born-WKBJ inversion method for acoustic reflection data: Geophysics, 46 (1981), 1559-1567.

[8] M. Jakobsen and B. Ursin, Nonlinear seismic waveform inversion using a Born iterative Tmatrix method. SEG Technical Program Expanded Abstracts 2012.

[9] M. Jakobsen and B. Ursin, Full waveform inversion in the frequency domain using direct iterative T-matrix methods. Journal of Geophysics and Engineering, 12 (2015), 400-418.

[10] M. Jakobsen and R. S. Wu, Accelerating the T-matrix approach to seismic full waveform inversion by domain decomposition. Geophysical Prospecting, 66 (2018), 1039-1059.

[11] R. E. Kleinman and P. M. van den Berg, A modified gradient method for two-dimensional problems in tomography. Journal of Computational and Applied Mathematics, 42 (1992), 17-35.

[12] P. Lailly, The seismic inverse problem as a sequence of before stack migrations: Conference on Inverse Scattering, Theory and Application, Society of Industrial and Applied Mathematics, Expanded Abstracts, 206-220, 1983.

[13] H. W. Levinson and V. A. Markel, Solution to the nonlinear inverse scattering problem by T-matrix completion. I. Theory. Physical Review E, 94 (2016), 043317.

[14] R. G. Pratt, Seismic waveform inversion in the frequency domain, Part 1: Theory and verification in a physical scale model. Geophysics, 64 (1999), 888-901. 
[15] R. G. Pratt, C. Shin, Hicks, Gauss-newton and full newton methods in frequency-space seismic waveform inversion. Geophysical Journal International, 133(1998), 341-362.

[16] C. Shin, Y. H. Cha, Waveform inversion in the Laplace domain. Geophysical Journal International. 173 (2008), 922-931.

[17] C. Shin, Y. H. Cha, Waveform inversion in the LaplaceFourier domain. Geophysical Journal International. 177 (2009), 1067-1079.

[18] A. Tarantola, Inversion of seismic reflection data in the acoustic approximation, Geophysics, 49 (1984), 1259-1256.

[19] P. M. van den Berg and R. E. Kleinman, A contrast source inversion method. Inverse problems, 13 (1997), 1607.

[20] J. Virieux, and S. Operto, An overview of full-waveform inversion in exploration geophysics. Geophysics, 74 (2009), WCC1-WCC26.

[21] Y. M. Wang and W. C. Chew, An iterative solution of the twodimensional electromagnetic inverse scattering problem. International Journal of Imaging Systems and Technology, 1(1989), 100-108.

[22] A. B. Weglein, F. V. Arajo, P. M. Carvalho, et al. TOPICAL REVIEW:, Inverse scattering series and seismic exploration. Inverse problems, 19(2003), R27-R83. 